\title{
Cowgotchi: un juego serio para la mejora de la motivación y el aprendizaje en alimentación animal
}

\author{
Francisco Maroto Molina ${ }^{a}$, José A. Adame Siles ${ }^{b}$, Cecilia Riccioli ${ }^{c}$, Ana Garrido Varo ${ }^{\mathrm{d}}$ y Dolores C. \\ Pérez Marín \\ Grupo Docente 44, Universidad de Córdoba, Campus de Rabanales, Ctra. Madrid-Cádiz km 396, 14014, Córdoba,

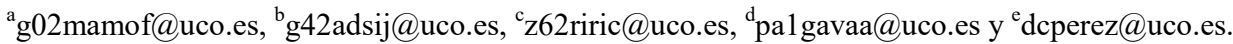

\begin{abstract}
The Teaching Group no. 44 of the University of Cordoba has extensive experience in incorporating innovative tools in teaching different subjects related to the area of Animal Production. This work describes the implementation of a new tool: gamification applied to animal rationing exercises. The objective of this new tool is to improve the motivation and learning of the students of the subject "Engineering and Technology for Animal Production". A serious game has been developed, in the form of an Android app, inspired by Bandai's Tamagotchi ${ }^{\circledR}$, a very popular game in the 2000s that was based on caring for and feeding a virtual pet to keep it alive and get different bonuses. In this case, the game is about the calculation of adequate rations to keep a dairy cow alive, take care of its health and maximize milk production while minimizing the cost of feeding. Current game functioning and the planned steps to improve the its characteristics of serious game are described.
\end{abstract}

Keywords: gamification, serious games, learning motivation, self-learning.

\section{Resumen}

El Grupo Docente 44 de la Universidad de Córdoba tiene una amplia experiencia en la incorporación de herramientas innovadoras en la docencia de distintas asignaturas relacionadas con el área de la Producción Animal. En este trabajo se describe la implementación de una nueva herramienta: la gamificación aplicada a los ejercicios de racionamiento animal. El objetivo de esta nueva herramienta es la mejora de la motivación y el aprendizaje de los estudiantes de la asignatura "Ingeniería y Tecnología de la Producción Animal". Se ha desarrollado un juego serio en forma de aplicación Android inspirado en el Tamagotchi ${ }^{\circledR}$ de Bandai, juego muy popular en los años 2000 que se basaba en cuidar y alimentar una mascota virtual para mantenerla viva y obtener diversas bonificaciones. En este caso se trata de calcular raciones adecuadas que permitan mantener viva una vaca lechera, cuidar su salud y maximizar la producción de leche minimizando el coste de la alimentación. Se describe el funcionamiento del juego y los siguientes pasos para mejorar el carácter de juego serio de la aplicación móvil desarrollada.

Palabras clave: gamificación, juegos serios, motivación al aprendizaje, autoaprendizaje. 


\section{Introducción}

Los profesores y demás personal del Grupo Docente 44 de la Universidad de Córdoba trabajan desde hace más de 15 años en la adaptación de sus metologías docentes a los lineamientos del Espacio Europeo de Educación Superior (EEES). Paulatinamente, han ido sustituyendo el modelo de docencia universitaria tradicional, basado casi exclusivamente en las clases magistrales, por una combinación de estas con clases interactivas, actividades fuera del aula, estudios de casos y proyectos. Todas estas herramientas se enmarcan en una línea de mejora docente basada en el autoaprendizaje (self-learning) y el aprender haciendo (learning-by-doing). Por otro lado, en tanto que las actividades docentes del Grupo se desarrollan en el campo de la Ingeniería Agrónomica, en concreto en el área de la Producción Animal, se ha hecho hincapié en acercar la docencia a los problemas reales del sector agroganadero.

La gamificación, en concreto los juegos serios (serious games), constituye una innovación docente que complementa perfectamente a las citadas anteriormente. Un juego serio es una prueba mental, de acuerdo con unas reglas específicas, que usa la diversión como modo de formación, con objetivos en el ámbito de la educación, la sanidad, la política pública y la comunicación estratégica (Zyda, 2005). Diversos autores (Prince, 2013; Brigham, 2015) destacan la motivación de los estudiantes como uno de los elementos clave de la gamificación, la cual está relacionada con aspectos cognitivos, afectivos, normativos y socioculturales que deben ser tenidos en cuenta en el diseño del juego para que éste logre su objetivo de aprendizaje.

La alimentación animal y, en concreto, el racionamiento, es uno de los campos más complejos de la producción animal, debido a los múltiples condicionantes que deben ser tenidos en cuenta: características de los alimentos, necesidades de los animales, costes de las materias primas, gestión de los stocks, producción de residuos... En la docencia tradicional sobre racionamiento animal, se enfrenta al estudiante a una serie de "problemas tipo" que, si bien le proporcionan los conocimientos relativos a los elementos básicos del racionamiento, no le permiten apreciar las consecuencias de sus decisiones en la respuesta animal, ya sean positivas (producción) o negativas (salud animal y contaminación ambiental). Por otro lado, este tipo de ejercicios son lejanos a la realidad práctica de la producción animal, lo cual resta motivación al alumnado. La gamificación es una oportunidad para superar estos problemas, en tanto que mejora la motivación de los estudiantes, al mantenerlos comprometidos con una actividad diseñada con elementos motivacionales durante un periodo prolongado de tiempo. Estos elementos incluyen sistemas de incentivos, como puntuaciones, tablas de clasificación y trofeos, así como otros mecanismos y actividades que los alumnos disfruten o encuentren interesantes, por ejemplo los elementos humorísticos (Hidi y Renninger, 2006; Rotgans y Schmidt, 2011). Otro argumento habitual a favor de la gamificación es que permite perder el "miedo a fallar". El estudiante, en lugar de concebir el fracaso como un resultado indeseable, lo considera como un paso esperado e incluso necesario en el proceso de aprendizaje (Kapur, 2006; Kapur y Bielaczyc, 2012). Las reducidas consecuencias del fracaso en el ámbito de un juego fomentan la toma de riesgos, probar cosas nuevas y la exploración (Hoffman y Nadelson, 2010).

\section{Objetivos}

El objetivo general del trabajo es el desarrollo de una práctica gamificada que permita mejorar la motivación del estudiantado de la asignatura "Ingeniería y Tecnología de la Producción Animal" del $2^{\circ}$ curso del "Grado en Ingeniería Agronómica y del Medio Rural" de la Universidad de Córdoba en lo referente al aprendizaje del racionamiento animal. 
Específicamente, se trata de desarrollar un juego serio con forma de aplicación móvil que simule el racionamiento de vacas lecheras e incorpore elementos de cálculo, de ensayo-error y de validación de los resultados obtenidos en diferentes ámbitos (producción, beneficio económico y salud animal) en base a las decisiones adoptadas por el estudiante, así como elementos de gamificación, como sistemas de puntaje y competencia entre pares.

\section{Desarrollo de la innovación}

\subsection{Diseño del juego}

Como se ha explicado, el juego se basa en la evaluación de la adecuación de una ración para vacas lecheras calculada por el estudiante en lo referente a salud animal, producción de leche y beneficio económico, lo cual se corresponde con algunas de las competencias que éstos deben adquirir en la asignatura "Ingeniería y Tecnología de la Producción Animal". En la bibliografía se pueden encontrar modelos diversos para el cálculo de la respuesta animal, con diverso grado de complejidad (Johnson y col., 2015). Algunos de esos modelos incluso han sido codificados en forma de herramientas informáticas, como MOLLY (Baldwin, 1995). Sin embargo, la mayor parte de estas herramientas carecen de los elementos lúdicos característicos de un juego, con lo que, en general, no proporcionan una mejora de la motivación de los estudiantes.

Teniendo en cuenta lo anterior, el diseño del juego objeto de este trabajo se ha inspirado en el Tamagotchi®, mascota virtual creada en 1996 por Aki Maita y comercializada por Bandai, que fue muy popular en los primeros años del siglo XXI. El Tamagotchi ${ }^{\circledR}$ debía ser cuidado y alimentado por el jugador para mantenerlo vivo. Además, el jugador obtenía puntos que le permitían conseguir accesorios diversos. En el juego objeto del presente trabajo, bautizado como Cowgotchi, el jugador (el estudiante) debe alimentar una vaca para que se mantenga viva y sana, y para que produzca la máxima cantidad de leche al menor coste posible. En lugar de puntos, el estudiante obtiene ingresos por la venta de la leche producida, de los cuales se descuentan los costes de la alimentación, y compite con sus compañeros de clase por obtener el máximo beneficio de la actividad ganadera. Cabe destacar que el juego simula una situación real, en la que un Ingeniero Agrónomo especializado en nutrición animal debe elaborar una dieta que permita cubrir las necesidades de los animales, maximizando al mismo tiempo el beneficio de los ganaderos, para lo cual debe elegir entre los alimentos disponibles en el mercado aquellos que, mezclados adecuadamente, maximicen la producción de leche con el menor coste.

Los datos de composición química y valor nutritivo de los alimentos y los datos de necesidades nutricionales de los animales, así como los modelos de respuesta animal sobre los que se construye el juego, se han obtenido de las tablas INRA (2007) y FEDNA (2010), por ser éstos los sistemas de alimentación explicados en la asignatura "Ingeniería y Tecnología de la Producción Animal".

Por otro lado, el juego se ha desarrollado íntegramente en inglés, en consonancia con el Plan para el Fomento del Plurilingüismo de la Universidad de Córdoba.

\subsection{Programación del juego}

Como primera aproximación, se ha construido un prototipo del juego en MS Excel ${ }$, con el objetivo de testar su funcionalidad en relación con los objetivos de aprendizaje. Los profesores y colaboradores firmantes de este trabajo han evaluado esta primera versión del juego y han propuesto diversas mejoras.

A continuación, se ha construido una aplicación móvil Android® nativa compatible con Android 5.0 y versiones posteriores que incorpora las mejoras propuestas. Como lenguaje de programación se ha usado 
Java ${ }^{\circledR}$. Para el almacenamiento y procesamiento de los datos (alimentos, usuarios, raciones...) se ha usado un servidor cloud basado en Apache ${ }^{\circledR}$ y MySQL $\AA$.

\section{Resultados}

En su versión actual, el juego dispone de una base de datos de 70 ingredientes diversos (cereales, subproductos de cereales, melazas y vinazas, concentrados de proteína vegetal, alimentos fibrosos, productos de origen animal, forrajes, subproductos fibrosos húmedos y otros) entre los cuales los estudiantes deben seleccionar de 1 a 6 para elaborar sus raciones.

Los estudiantes deben calcular una ración diaria durante un periodo de tiempo establecido por los profesores (de 5 a 7 días). El cálculo de estas raciones debe ser manual, con lo cual se incentiva la práctica por parte del estudiantado de uno de los ejercicios clave de la asignatura "Ingeniería y Tecnología de la Producción Animal”, el cual representa una parte signficativa del examen de evaluación. El estudiante puede elegir utilizar la misma ración cada uno de los días de duración del juego, pero todas las raciones utilizadas por cada estudiante quedan registradas en la base de datos y son accesibles al profesorado, que de este modo puede evaluar el nivel de involucración de cada estudiante.

Con base en los modelos matemáticos incluidos en el juego, cada dieta genera una respuesta animal en forma de mantenimiento de la salud de la vaca y de producción de leche, de tal modo que si no estuviera bien calculada la producción disminuye e incluso se anula. Cada día el juego calcula el beneficio, o déficit, resultante de restar el coste de la alimentación de los ingresos por venta de leche, siendo el ganador del juego aquel estudiante que al final del mismo dispone de un mayor saldo. Para formentar la competencia entre pares, los estudiantes pueden consultar cada día el ranking provisional.

Por otro lado, de cara a favorecer el aprendizaje, el juego proporciona un feedback al estudiante cuando la ración no está bien calculada, indicándole cuál o cuáles son los problemas. Se muestran los siguientes mensajes, acompañados de una imagen humorística para mejorar la jugabilidad:

- "I cannot survive on this" cuando las necesidades de conservación en términos de energía y/o proteína (las que necesita la vaca para mantenerse viva) no se cubren con la ración elaborada.

- "I am able to produce milk, but you can feed me better" cuando se cubren las necesidades de conservación, pero la energía y/o proteína para producción de leche están por debajo del potencial productivo de la vaca.

- "A lot of milk today, congratulations!" cuando se cubren las necesidades de conservación y se alcanza el máximo productivo.

- "I am losing nitrogen in urine" cuando existe un exceso de proteína en el rumen (PDIN > PDIE + $20 \%)$.

- "Have you considered using urea?" cuando existe un exceso de energía en el rumen (PDIE > PDIN + 20\%)

- "I am a ruminant and I need fiber" cuando la fibra total de la ración es inferior al 15\%, necesario para mantener la salud ruminal de la vaca.

- "I have stomachache, too many concentrates" cuando la proporción de forrajes en la ración es inferior al 60\%, necesario para mantener la salud ruminal de la vaca.

- "Too much urea, it is poisoning me" cuando la urea representa más del $1 \%$ de la ración, lo cual resulta tóxico para el animal. 
La figura 1 recoge el diagrama de flujo que resume el funcionamiento del juego:

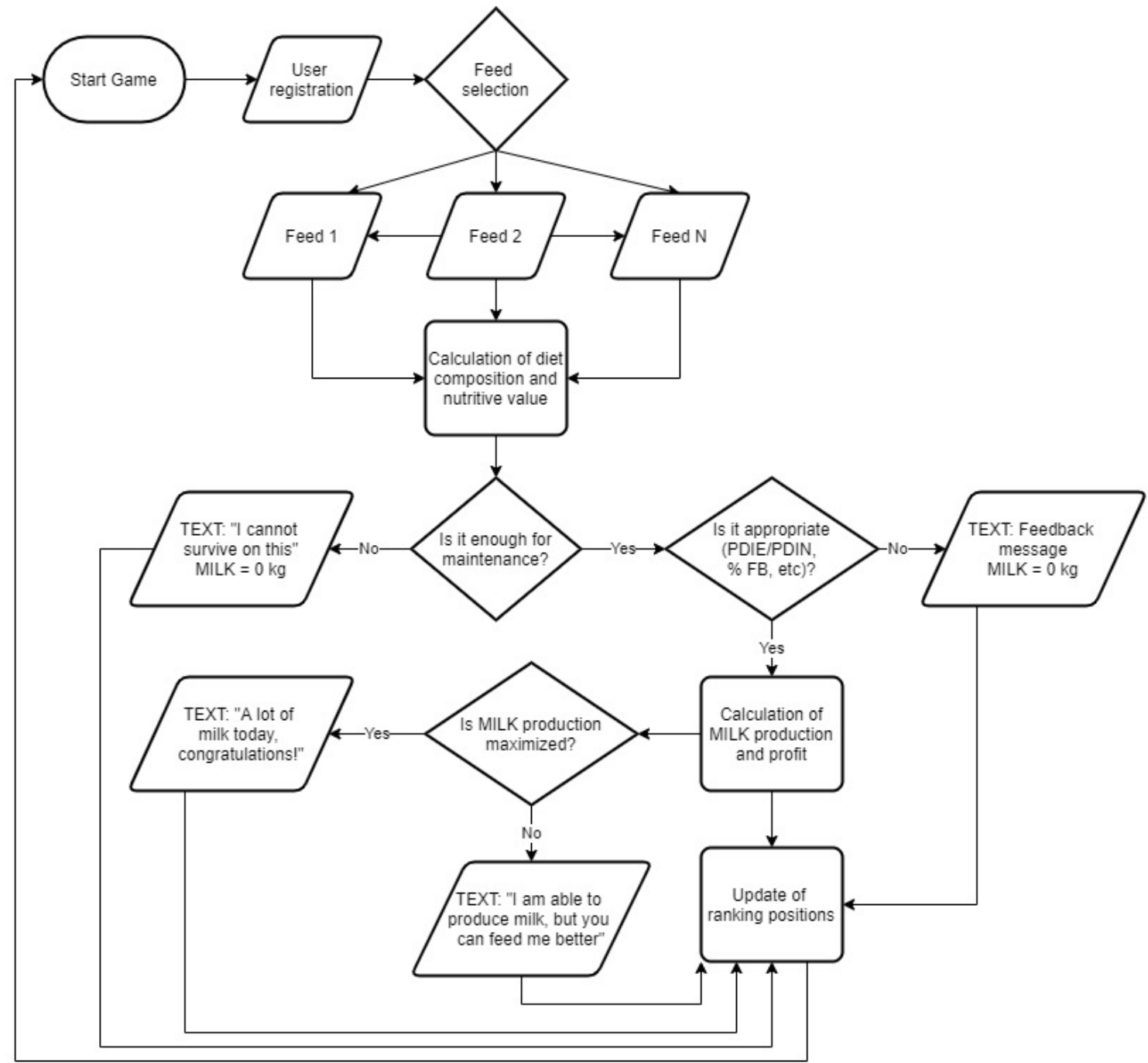

Fig. 1 Diagrama de flujo del juego.

A continuación se recogen las pantallas que componen la aplicación móvil con el fin de mostrar la estética del juego y la incorporación de elementos humorísticos en dicha estética. 

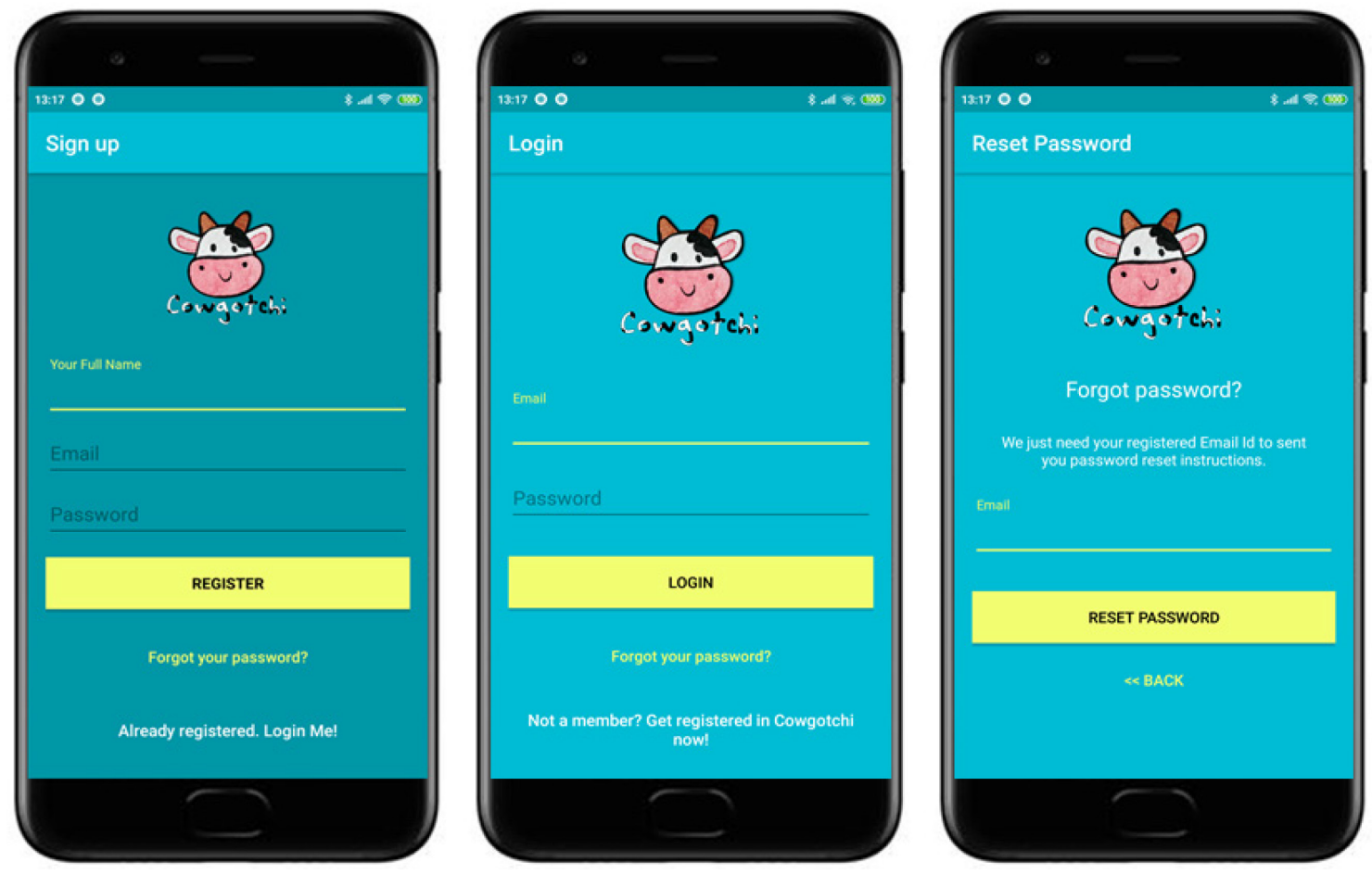

Fig. 2 Registro y login.
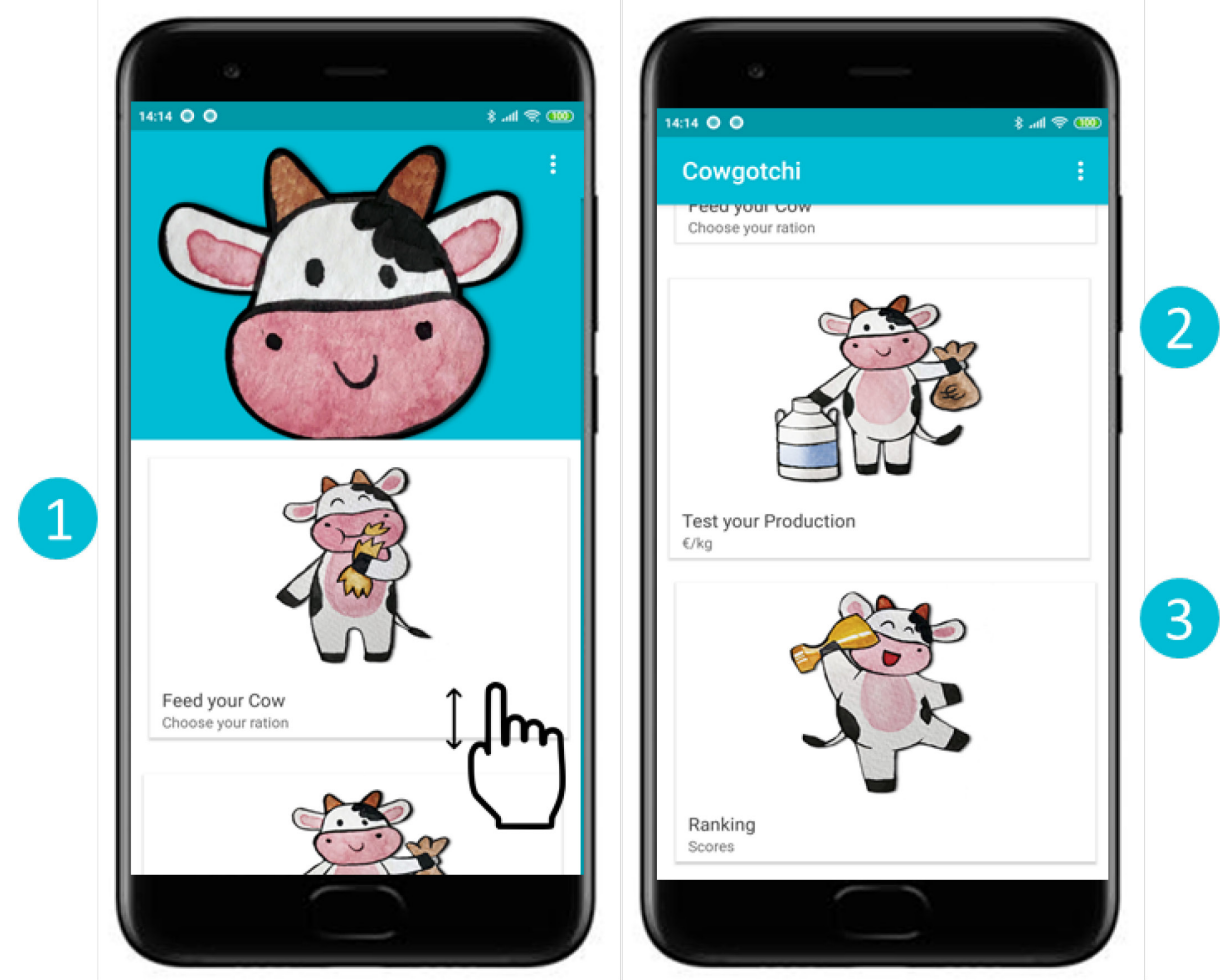

Fig. 3 Menú principal. 

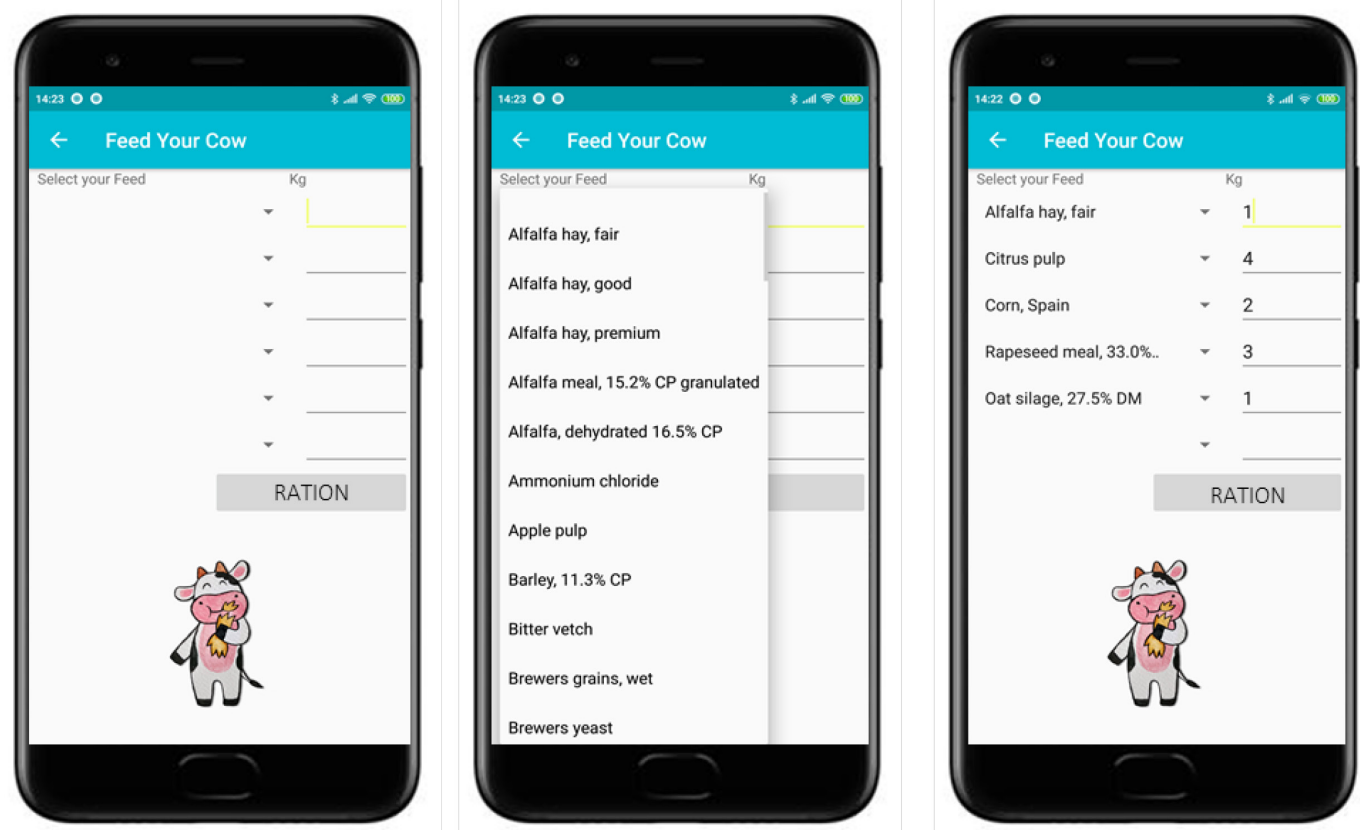

Fig. 4 Selección de alimentos.
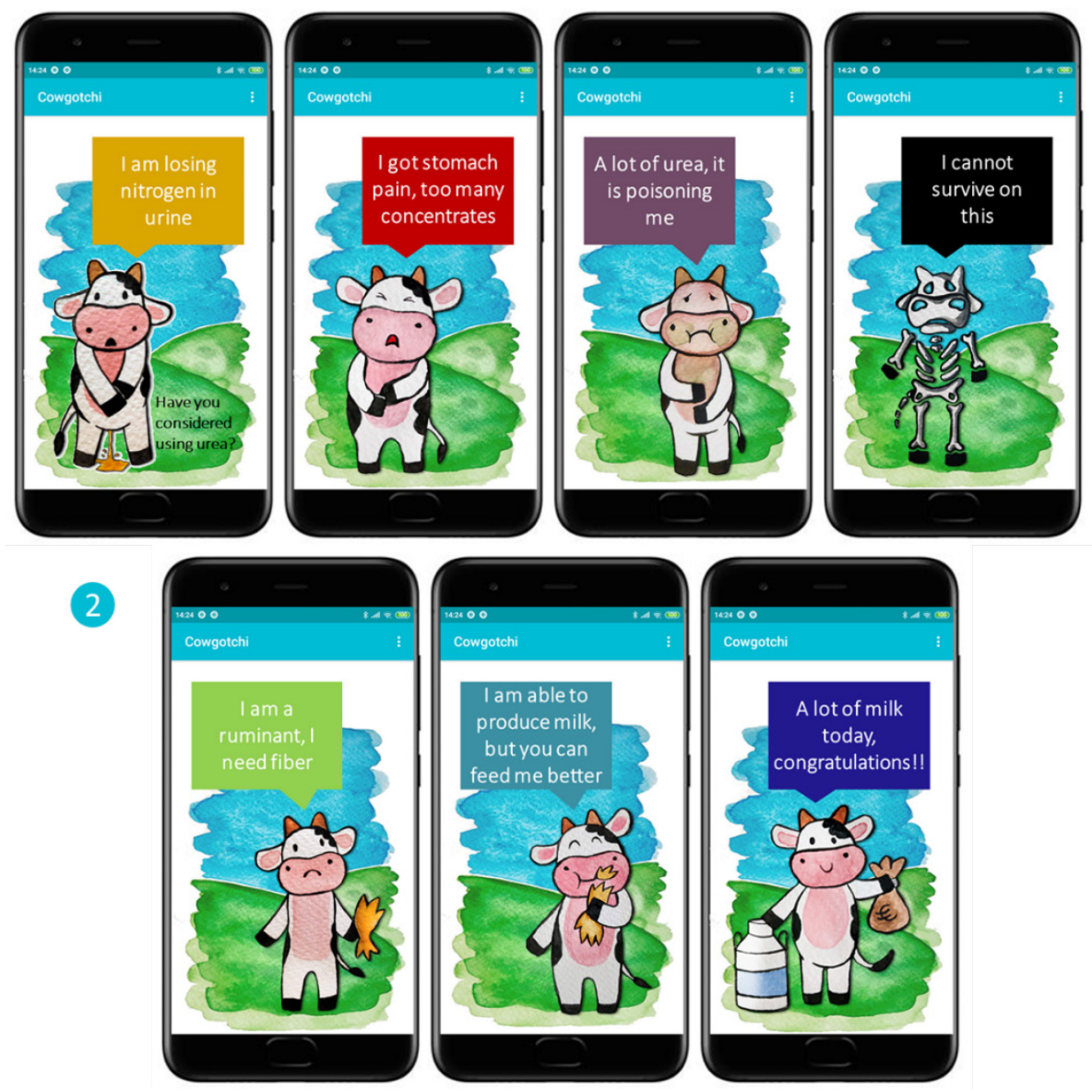

Fig. 5 Mensajes de feedback. 


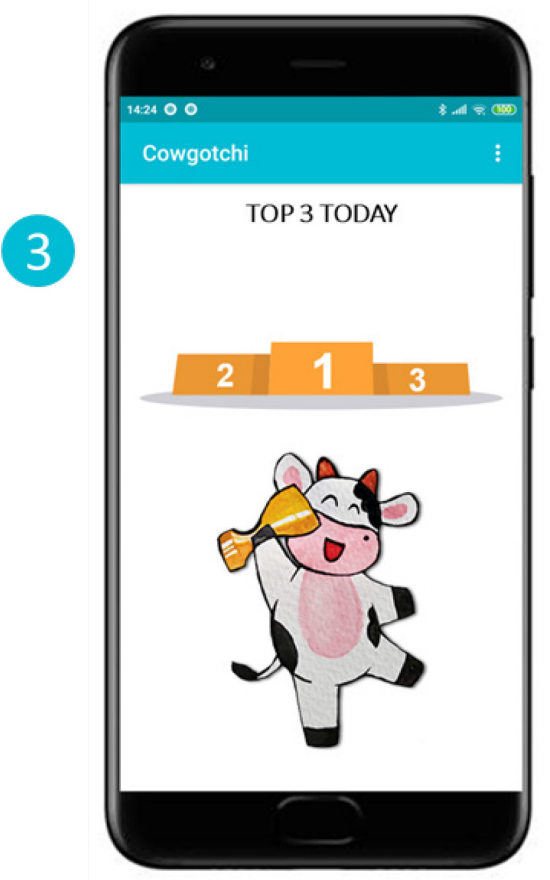

Fig. 6. Ranking de los estudiantes.

\section{Conclusiones y consideraciones futuras}

El juego Cowgotchi consigue la gamificación de un ejercicio de racionamiento de vacas lecheras, lo cual permitirá mejorar la motivación de los estudiantes respecto al aprendizaje de la alimentación animal, y, en particular, su predisposición a practicar los ejercicios de cálculo de raciones fuera del aula.

Parte de la mejora de la motivación se debe a que el juego simula situaciones cercanas a la realidad profesional que van a encontrar los futuros egresados. En este sentido, el Grupo Docente 44 ya está trabajando en incorporar nuevas funciones al juego que permitan acercarlo aún más a la realidad productiva, lo cual supone mejorar su carácter de juego serio. Entre las funciones que se están incorporando en Cowgotchi 2.0 cabe destacar las siguientes:

- Cada estudiante dispondrá al inicio del juego de un rebaño de 100 vacas, en lugar de una vaca individual.

- Los estudiantes podrán utilizar los beneficios obtenidos de la venta de leche para aumentar el tamaño de su rebaño, lo cual les permitirá evaluar decisiones empresariales no estrictamente relacionadas con la práctica del racionamiento animal.

- Se limitará la cantidad disponible de cada alimento, de tal modo que los estudiantes deberán competir por los alimentos más convenientes tanto desde el punto de vista nutricional como económico. Esta nueva función evitará que los estudiantes se limiten a usar los alimentos más habituales y calculen solo raciones "clásicas". Además, los obligará a incorporar elementos de estrategia en su modo de juego, por ejemplo apostando al principio del juego por aquellos alimentos más interesantes de los cuales se prevé escasez en los días siguientes. 


\section{Referencias}

BALDWIN, R.L. (1995). Modelling Ruminant Digestion and Metabolism. Londres. Editorial Chapman and Hall.

BRIGHAM, T.J. (2015). “An Introduction to Gamification: Adding Game Elements for Engagement” en Medical Reference Services Quarterly, vol. 34, issue 4, p. 471-480.

FEDNA (2010). Tablas FEDNA de composición y valor nutritivo de alimentos para la fabricación de piensos compuestos ( $3^{a}$ edición). Madrid. Editorial FEDNA.

HIDI, S. y RENNIGER, K.A. (2006). "The four-phase model of interest development” en Educational Psychologist, vol. 41, issue. 2, p. 111-127.

HOFFMAN, B. y NADELSON, L (2010). "Motivational engagement and video gaming: A mixed methods study" en Educational Technology Research and Development, vol. 58, issue. 3, p. 245-270.

INRA (2007). Alimentación de bovinos, ovinos y caprinos. Necesidades de los animales. Valores de los alimentos. Madrid. Editorial Quae.

JOHNSON, I.R., FRANCE, J. y CULLEN, B.R. (2015). “A model of milk production in lactating dairy cows in relation to energy and nitrogen dynamics” en Journal of Dairy Science, vol. 99, p. 1605-1618.

KAPUR, M. (2006). "Productive failure”. Barab, S., Hay, K. y Hickey, D. (coords.). En: Proceedings of the 7th international conference on Learning. Bloomington: Indiana. 307-313.

KAPUR, M. y BIELACZYC, K. (2012). "Designing for Productive Failure” en Journal of the Learning Sciences, vol. 21 , issue. 1 , p. $45-83$.

PRINCE, J.D. (2013). “Gamification” en Journal of Electronic Resources in Medical Libraries, vol. 10, issue 3, p. 162-169.

ROTGANS, J.I. y SCHMIDT, H.G. (2011). "Situational interest and academic achievement in the active-learning classroom" en Learning and Instruction, vol. 21, issue 1, p. 58-67.

ZYDA, M. (2005). "From visual simulation to virtual reality to games" en Computer, vol. 38, issue 9, p. 25-32. 